\title{
Cartografía histórica
}

\section{y reconstrucción paleotopográfica y geoarqueológica de la ciudad romana de Caesar Augusta (Zaragoza, España)}

\author{
L.A. Longares Aladrén ${ }^{(1)}$, J.L. Peña-Monné(1), F. Escudero Escudero ${ }^{(2)}$ y F. Pérez-Lambán ${ }^{(3)}$ \\ (1) Departamento de Geografía y Ordenación del Territorio, Facultad de Filosofía y Letras, Universidad de Zaragoza, \\ Calle Pedro Cerbuna s/n, E-50009 Zaragoza, España \\ lalongar@unizar.es, jlpena@unizar.es \\ (2) Servicio de Cultura, Educación y Medio Ambiente, Ayuntamiento de Zaragoza, Vía Hispanidad, 20 Planta $1^{\mathrm{a}}$, \\ E-50071 Zaragoza, España \\ 9762105393@telefonica.net \\ (3) Departamento de Ciencias de la Antigüedad, Facultad de Filosofía y Letras, Universidad de Zaragoza, \\ Calle Pedro Cerbuna s/n, E-50009 Zaragoza, España \\ fperezlamban@gmail.com
}

\begin{abstract}
RESUMEN
En este trabajo se ofrece una aproximación a la reconstrucción de la topografía y las características del terreno de Caesar Augusta (Zaragoza). Esta reconstrucción ha sido modelada integrando en un Sistema de Información Geográfico (SIG) el mapa topográfico de Casañal de 1880 -anterior a las principales transformaciones urbanas ocurridas en la ciudad- e información geomorfológica, geoarqueológica y arqueológica. Caesar Augusta fue fundada por los romanos junto al río Ebro en el siglo I aC sobre un asentamiento ibérico previo (Salduie). El área urbana ocupaba una terraza fluvial cuaternaria en la confluencia de los ríos Ebro y Huerva. Esta terraza se eleva unos $10 \mathrm{~m}$ sobre esos ríos, con altitudes que varían entre 200-208 m s.n.m. La topografía actual de la ciudad ha variado sustancialmente a lo largo del tiempo, de modo que no es apropiada para reconstruir la morfología original del terreno donde los romanos fundaron Caesar Augusta. Se ha utilizado ArcGIS 10.0 y sus módulos ArcMap y ArcScene para la gestión y análisis de los datos. Los modelos 3D generados muestran muchos elementos topográficos de detalle que en mapas anteriores pasaban desapercibidos. Estos modelos son complementados con datos de la naturaleza geológica del subsuelo provenientes de observaciones realizadas en diversas excavaciones arqueológicas. El modelo digital del terreno derivado del mapa de Casañal y las informaciones complementarias sólo son válidas para reconstrucciones generales. Sin embargo, ha mejorado nuestro conocimiento sobre muchos elementos romanos de Caesar Augusta (topografía del Decumanus y Cardo maximus, cloacas, fosos, muralla, teatro y foro). Y, es más, es ahora posible comprender mejor la geomorfología y el dinamismo fluvial de la ciudad de Zaragoza.
\end{abstract}

Palabras clave: depresión del Ebro, Geoarqueología, Geomorfología, modelos digitales de terreno, terraza fluvial.

\section{Historical mapping and geoarchaeological reconstruction of the Roman city of Caesaraugusta (Zaragoza, Spain)}

\begin{abstract}
In this paper we provide a reconstruction of the topography and characteristics of the terrain of Caesar Augusta (the Roman name of the present-day city of Zaragoza). This reconstruction has been modelled using GIS and integrating the topographic map drawn by Casañal in 1880 -previous to the main urban transformations- with geomorphological, geoarchaeological and archaeological data. Caesar Augusta was founded by Romans near the Ebro River in the $1^{\text {st }}$ century $B C$ in the same place where there was a previous Iberian settlement (Salduie). The urban area occupied a quaternary fluvial terrace in the confluence of the rivers Ebro and Huerva. The terrace stands about $10 \mathrm{~m}$ above these rivers, with a varying height between 208-200 masl.
\end{abstract}


Longares Aladrén, L.A., et al., 2018. Cartografía histórica y reconstrucción... Boletín Geológico y Minero, 129 (1/2): $421-435$

The present-day urban topography has suffered many transformations throughout history; therefore, it is impossible to reconstruct the original morphology of the land where the Romans decided to build their town in the past. We have used ArcGIS Desktop 10.0 and its platforms ArcMap and ArcScene for managing and analysing the data. 3D models show many fine topographic features that in were overlooked previous maps. These models are complemented with information about the underground geologic nature, which is known through the observations made in several archaeological excavations. The model derived from Casañal s map and complementary data is only valid for general reconstructions, but it has enhanced our knowledge of many elements of Caesar Augusta (topography of the Decumanus and Cardo maximus, sewage system, moats, wall, theatre, forum), making it possible to better understand the geomorphology and fluvial evolution of the city of Zaragoza.

Key words: Geomorphology, Geoarchaeology, elevation digital models, Ebro basin, fluvial terrace.

\section{ABRIDGED ENGLISH VERSION}

\section{Introduction and methods}

Zaragoza is situated in the central Ebro Basin (NE Spain), in a Mediterranean biogeographic and climatic region, with important continental influence. The geological substratum is formed by evaporitic formations of the semi-arid environments in the distal part of a Tertiary endorheic basin. The Miocene gypsum of the Zaragoza formation is the main lithology and it is demarcated in its margins by structural platforms made of limestone from the end of the Miocene. These platforms have been formed by the intense incision caused by the Quaternary fluvial network. In Zaragoza, the Miocene gypsum is covered by thick Quaternary terrace accumulations deposited by the rivers Ebro, Huerva and Gállego. The particular behaviour of the gypsum has created a nested fluvial terrace system, with local deformation and thickening due to the karstic processes of the underlying gypsum. As a result, the old terraces of this system are located near the present rivers; the terrace on top of which Zaragoza is built is 7-9 $\mathrm{m}$ above the current riverbed and it is formed by cemented gravels. This terrace was formed during one of the intense fluvial accumulation phases of the Quaternary, which was dated in the river Gállego in 156-181 ky BP. The thickness of this terrace prevents the underlying gypsum to surface in the urban area of Zaragoza. On the other hand, a Holocene terrace covers the northern bank of the river Ebro and the north-eastern part of the Roman city. Sands from the rivers Ebro and Huerva have been recovered from the archaeological excavations, and, in some cases, dated to Roman times.

Most Roman cities throughout the whole Mediterranean region have been populated continuously until the present, making the reconstruction of its original topography difficult. This is the case of Caesar Augusta, located under the historical city centre of the present-day city of Zaragoza. This Roman city was founded in the place previously occupied by an Iberian settlement called Salduie, near the confluence of the rivers Ebro, Huerva and Gállego, in the central Ebro Basin (Fig. 1). The settlement was fortified by a wall, whose defensive function prevailed during the Middle Ages under Islamic and Christian domination. Until the second half of the $19^{\text {th }}$ Century, the city remained within this enclosure (Fig. 2), which defines the historical city centre where many archaeological remains and stretches of wall are preserved (Fig. 3).

In this paper we provide data for a reconstruction of the topography and characteristics of the terrain of Caesaraugusta through two different approaches:

Firstly, the topographic map drawn in 1880 by the engineer Dionisio Casañal Zapatero with triangulation methods. This map represents the city of Zaragoza in a 1:1000 scale with $0.5 \mathrm{~m}$ interval between contour lines.

Secondly, stratigraphic data and archaeological information recovered from urban archaeological excavations in the Roman city area. On the one hand, these data provide level references for Roman buildings sewers and pavements and on the other hand they give geoarchaeological context to some important monuments, such as the Roman city.

In this study we used Esri's ArcGIS Desktop 10.0 and its platforms ArcMap and ArcScene for managing and analysing the data. A high resolution image (1200 ppp, divided into 20 parts) of Casañal s map was provided by the Town Hall of Zaragoza (Fig. 4) and conveniently incorporated to a GIS geo-database and geo-referenced by means of a minimum of 10 coincident ground control points (GCP) between each single map part (origin) and a 2012 orthophotography (ETRS89, zone 30 north). When necessary, more GCPs were added and in all cases root-mean-square error (RMSE) turned out to be close to zero.

All contour lines were then digitalized into a vector layer with altitude equidistance of $0.5 \mathrm{~m}$. A triangulated irregular network (TIN) and the corresponding digital elevation model (DEM) were derived from these contour lines. Altitude levels from Roman floors excavated in several places of Zaragoza were used to validate the resulting model. This DEM was completed with data from archaeological excavations of Roman monuments (stretches of wall, theatre, forum, baths and the sewer system). 
Longares Aladrén, L.A., et al., 2018. Cartografía histórica y reconstrucción... Boletín Geológico y Minero, 129 (1/2): $421-435$

All this information was synthesised in a $3 D$ visualization and topographic profiles of the surface of the ancient Caesar Augusta and its surroundings. The DEM of Caesar Augusta was compared to a DEM of Zaragoza derived from LiDAR data (MDT5 IGN).

\section{Results and conclusions}

The map of the variation of altitude (Figs. 5, 6) shows most of the soil losses within the Roman urban area, especially in its eastern part. These altitude changes led to a decrease in topographical roughness. Elevations and valleys with NNE-SSW orientation are apparent in the 1880 model (Figs. 6, 7), but they are widely flattened nowadays. Some of the main streets in the historical centre of Zaragoza run through these valleys. On the other hand, the elevated areas are formed by Quarternary detritic lithologies (cemented old gravels) where the Romans laid the foundations for some of their buildings.

The Decumanus maximus runs WNW-ESE (Figs. 8A, $8 \mathrm{C}$ ), tangent to the general direction of the elevations and valleys. The construction of this street involved some flattening leaving a wavy topography as shown in Casañal s map.

The gradient between the elevated areas and the valleys reaches $4 \mathrm{~m}$ between the border of the Roman city and the valley at the foot of the wall (Figs. $8 \mathrm{~A}, 8 \mathrm{C}$ ). The groove of this valley continues after a $90^{\circ}$ turn along Coso street. As a result, the Roman city and wall are enclosed by a hypothetical defensive moat.

The cavea of the Roman theatre is laid on a number of elevations with sandy deposits. They are $3 \mathrm{~m}$ higher than Coso Street, which may support the hypothesis of the existence of a defensive moat (Fig. 8D).

In late Roman times, the city was enlarged beyond the walls, in the eastern area near the river Huerva. Here there is another elevation (2-2.5 $\mathrm{m}$ above Coso Street) with a stepped slope that connects with a low fluvial terrace of the river Huerva (Fig. 8B). Its gradient was used by the sewer system in this area, which drained towards the Huerva in this sector, while all the other sewers of the city drain to the Ebro River.

The topographic profile of the Decumanus IX (Fig. 9a) according to the DEM derived from Casañal s map shows the wavy trajectory of the street and the position of the Roman and Medieval walls, with the groove of the moat between them. It could also be argued that this groove was originally an ancient riverbed of the river Huerva, which deviated in Roman times.

A second profile (Fig. 9b) running through the Roman theatre and forum shows the groove of the moat at the foot of the wall, the adaptation of the theatre to an elevation and the forum to the gradient between the old and young terraces of the Ebro river.

Geomorphological and archaeological data inform us about the three fluvial sedimentary units in the area of Caesar Augusta (Fig. 10). Above $200 \mathrm{~m}$ a.s.l. there are fluvial sands on top of an old gravel terrace, analogous to those of the Gállego river dated at 150-180 ky BP. At a lower level there are recent gravels deposited after $100 A D$ forming a terrace that was occupied in Roman times and that suffered river floods.

The fluvial morphology of the Ebro and Huerva rivers has also undergone some changes. The Ebro river has remained more or less in the same position, besides a wider inundation bed and higher fluvial dynamism. Several floods in the $13^{\text {th }}$ and $14^{\text {th }}$ centuries affected the western sector of the city but it was moved back artificially to its normal position. The Huerva river borders the city through a deep canyon ( $7 \mathrm{~m}$ deep, $5 \mathrm{~m}$ wide). This is due to the incision processes that prevailed after Roman times, cutting Holocene deposits accumulated as a result of the erosion of the lower Huerva valley from the Neolithic to the Roman era due to anthropic deforestation. As has already been stated, it is possible that the final part of the riverbed deviated in Roman times. There are marsh sediments from the Huerva river under the Roman wall which were dated from $14 \mathrm{C}$ to the Bronze Age. In addition, the amphorae field created in Roman times to elevate the terrain and expand the city could have pushed the river to its current position.

\section{Introducción}

La reconstrucción de la morfología original de áreas arqueológicas situadas bajo zonas urbanas actuales plantea numerosas dificultades. Muchos de estos espacios han sido objeto de ocupación durante amplios periodos históricos y aunque inicialmente los primeros asentamientos, por lo general, buscaron la adaptación y aprovechamiento de la topografía existente, con el paso del tiempo y la superposición de ocupaciones urbanas sucesivas, con necesidad de nuevos espacios, construcción de sistemas defensivos, etc., derivaron hacia una progresiva transformación del ámbito de ocupación, modificando el relieve de los primeros asentamientos.

Este es el caso de numerosas ciudades romanas de todo el ámbito mediterráneo, entre ellas el asentamiento romano de Caesar Augusta, en el centro histórico de la actual Zaragoza. La ciudad se funda sobre el pequeño núcleo ibérico de Salduie y su espacio de 
ocupación se ha visto sometido desde el primer momento a alteraciones topográficas del terreno original para adaptarlo a un nuevo plano romano rodeado por un recinto amurallado. Algunas zonas fueron niveladas mediante muros y rellenos de sedimentos para conseguir una topografía más regular y plana en su trazado urbano y adaptar su red de saneamientos (cloacas), otros sectores tuvieron que ser saneados para evitar la entrada de humedad e incluso la posibilidad de inundación fluvial y algunas zonas serán utilizadas para ampliar la zona urbana hacia los ríos circundantes, mediante la elevación del terreno. Posteriormente a la época romana la ciudad medieval (Saraqusta) volverá a superponerse a la romana, con nuevas intervenciones que alterarán la topografía urbana.

Sin embargo, los grandes cambios topográficos en este espacio del casco antiguo de la ciudad y su entorno próximo se produjeron en la segunda mitad del siglo XIX, motivados por el crecimiento demográfico, industrial y comercial de Zaragoza (Clemente Fernández, 1996). Hasta la mitad del siglo XIX, la ciudad de Zaragoza se localiza en el recinto amurallado, cerrado por ocho puertas (Germán Zubero, 1996) y es a partir de la llegada del ferrocarril y el crecimiento de las instalaciones industriales en el entorno cuando se inicia la expansión de la ciudad y las grandes operaciones urbanísticas de esa época (Faus Pujol, 1978; Clemente Fernández, 1996). Son estos cambios recientes los que de forma más importante dificultan la reconstrucción del relieve sobre el que se asentó la primitiva ciudad romana. Son numerosas las modificaciones que se introducen en el plano urbano dentro del ámbito de la ciudad antigua, que en términos generales han derivado en una topografía más llana y con importantes cambios viarios, como el de la calle Alfonso I en 1866/1867.

Todas estas actuaciones conllevan nuevas alineaciones de las construcciones y en el trazado de las calles y aunque se adivina el trazado de época romana, la actual configuración urbana y, por supuesto, la topografía sólo pueden tomarse como referencia muy general respecto a la que sirvió de cimentación para la construcción de Caesar Augusta (Escudero and Galve, 2013). No podemos asumir, como ocurre con algunos modelos desarrollados hasta el momento, que los cambios del medio acaecidos desde época antigua no han resultado significativos (Orengo et al., 2011).

En este trabajo se aportan datos para aproximarse a una reconstrucción de algunos elementos de la paleotopografía de Caesar Augusta partiendo de dos vías de trabajo:

a) por una parte, la utilización del mapa topográfi- co realizado en 1880 por el ingeniero topógrafo Dionisio Casañal Zapatero, a escala 1:1000 y con equidistancia entre curvas de nivel de 0,5 metros. Este documento aporta una topografía detallada y de gran precisión al utilizar técnicas modernas de triangulación en su época, como se ha podido comprobar no sólo en la ciudad de Zaragoza, sino en otras de las que este autor elaboró levantamientos topográficos, como Vitoria, Pamplona, Córdoba y Huesca (Villanova Valero, 2011), ya que sus trabajos han sido utilizados para el planeamiento urbanístico hasta casi finales del siglo XX (Betrán and Serrano, 2014) y, desde su puesta en conocimiento para los arqueólogos, ha servido como base para la ubicación de los restos y excavaciones en la ciudad de Zaragoza (Álvarez Gracia, 1985). Este trabajo y la calidad que de él se desprende, han permitido reconstruir algunos rasgos de la morfología original de época romana, con anterioridad a las drásticas transformaciones que se realizaron después de su elaboración.

b) por otra parte, la información estratigráfica y las referencias obtenidas en algunas de las intervenciones arqueológicas efectuadas en la ciudad romana, que puede aportar cotas de los niveles fundamentales de edificios, cloacas o pavimentos (Escudero and Galve, 2014) y de algunos trabajos geoarqueólogicos desarrollados en relación con la muralla romana (Peña-Monné et al., 2009).

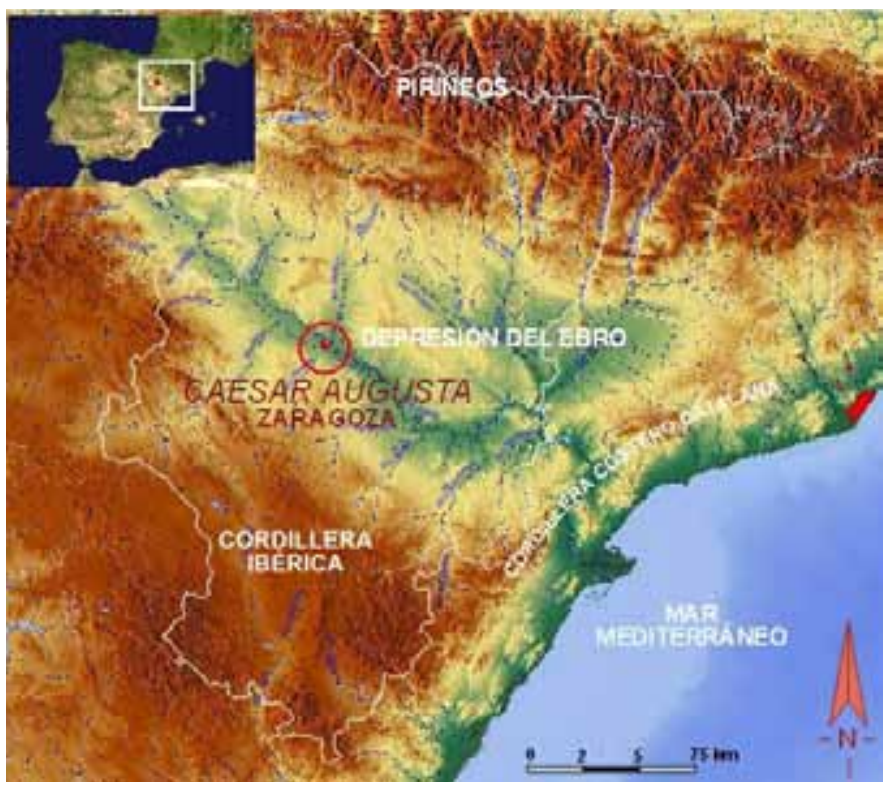

Figura 1. Localización de Caesar Augusta (actual Zaragoza) en el sector central de la Depresión del Ebro.

Figure 1. Location of Caesar Augusta (present-day Zaragoza) in the central sector of the Ebro basin. 


\section{Zona de estudio}

La ciudad romana de Caesar Augusta se asentó en la zona de confluencia de los ríos Ebro, Huerva y Gállego, en el sector central de la Depresión del Ebro (Fig. 1), ocupando parcialmente la anterior ciudad ibérica de Salduie. El recinto urbano quedaba delimitado por una muralla, cuya función defensiva continuó en época medieval, en la ciudad musulmana de Saraqusta y, posteriormente, en la ocupación cristiana, y solamente comenzará a expandirse fuera de este recinto a partir de mediados del siglo XIX (Fig. 2). En la actualidad este espacio constituye el casco histórico de la ciudad de Zaragoza, aunque se conservan numerosos tramos de la muralla romana, con importantes modificaciones durante el siglo XI (Paz Peralta, 2015) y restos arqueológicos de diferentes épocas (Fig. 3). La ciudad ha sido objeto de numerosos estudios históricos y arqueológicos, pero dado que el objetivo del trabajo se centra en aspectos reconstructivos a partir de los datos cartográficos y geomorfológicos, sólo citaremos a nivel orientativo algunas síntesis aportadas por Arce (1979), Lostal and Ansón (2001), Galve (2004), Beltrán Lloris and Fatás (1998), Beltrán Lloris (2007), Fatás (2008), Escudero and Galve (2013) que pueden ilustrar la importancia de la ocupación urbana en época Romana.

El sector central de la Depresión del Ebro presenta un sustrato geológico compuesto principalmente por formaciones evaporíticas correspondientes a ambientes sedimentarios de la zona distal de una cuenca endorreica terciaria. En el entorno de la ciudad los yesos del Mioceno de la Formación Zaragoza (Quirantes, 1978) constituyen las litologías dominantes, marginados al sur por relieves de plataformas estructurales calcáreas (muelas) fini-miocenas, como consecuencia de la intensa disección generada por la red fluvial cuaternaria. Las formaciones yesíferas

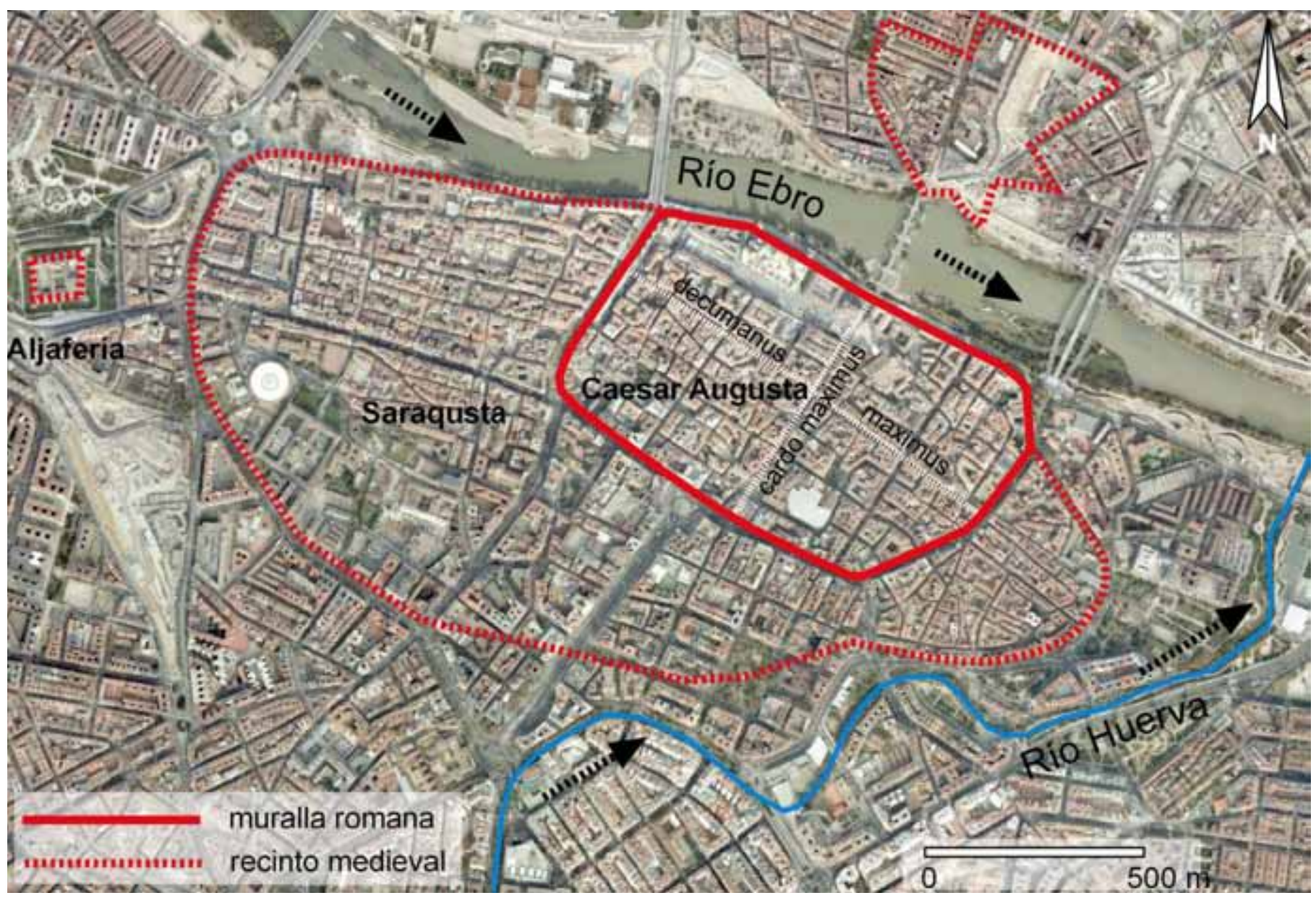

Figura 2. Esquema de las diferentes áreas de ocupación de la ciudad romana (Caesar Augusta) y medieval (Saraqusta).

Figure 2. Scheme of the different urban areas of the roman Caesar Augusta and medieval Saraqusta. 

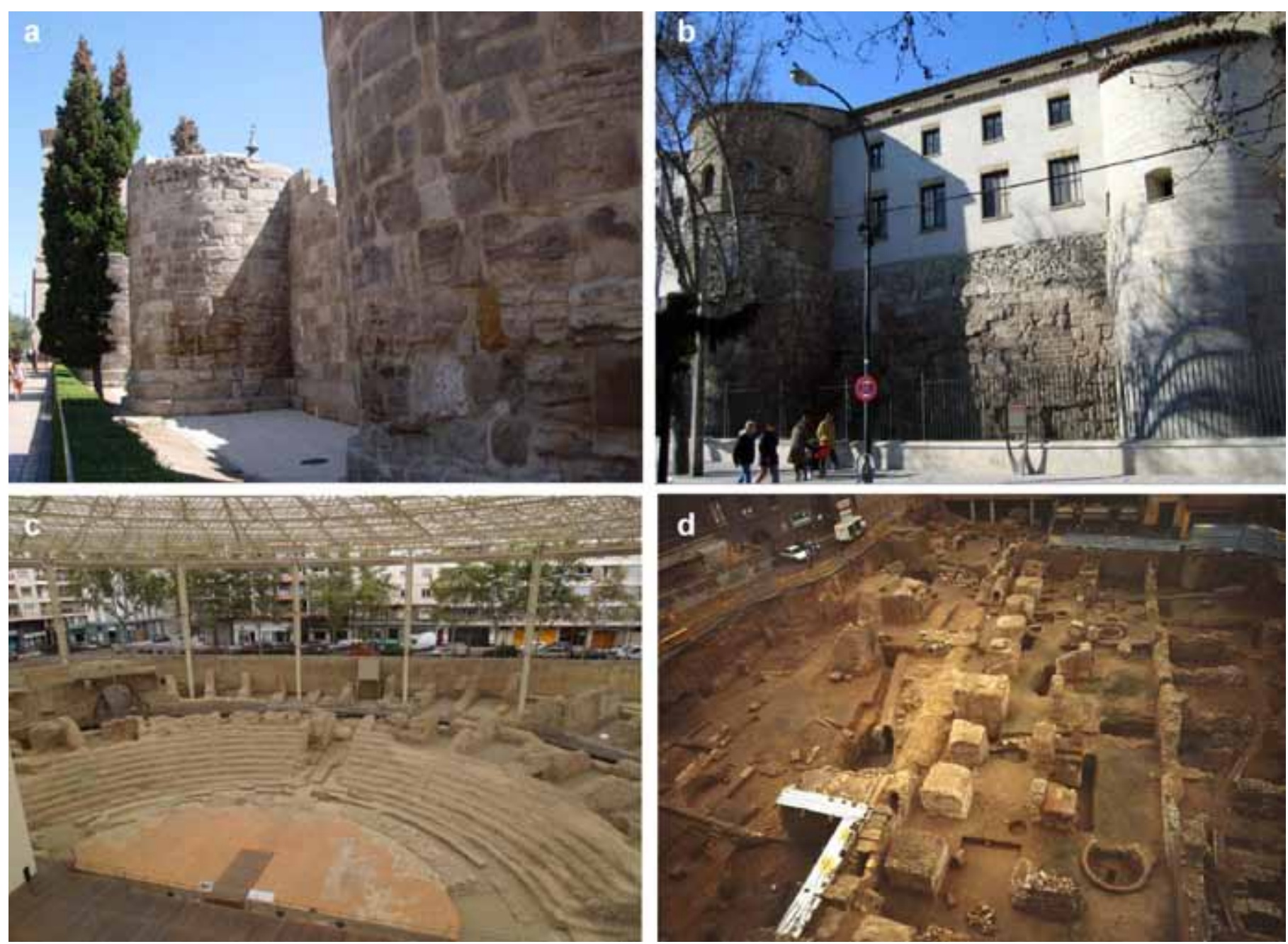

Figura 3. Elementos constructivos romanos más importantes de Caesar Augusta. a) Muralla de la Zuda; b) Muralla del Santo Sepulcro; c) Teatro romano tras su excavación; d) Excavaciones del Foro romano.

Figure 3. Most important Roman constructive elements of Caesar Augusta. a) Wall of Zuda; b) wall of the Holy Sepulchre; c) Roman theatre after excavation; d) excavations of the Roman Forum.

aparecen recubiertas por importantes acumulaciones de terrazas cuaternarias, depositadas por los ríos Gállego, Huerva y Ebro. El particular comportamiento de los yesos ha generado un sistema de terrazas fluviales encajadas, afectadas por procesos kársticos, que se manifiestan en engrosamientos y deformaciones de dichos depósitos (Benito et al., 1998). Por esta razón, las terrazas antiguas afloran en posiciones muy próximas a los ríos actuales. De tal manera que la terraza sobre la que se asienta la ciudad de Zaragoza, cuya superficie está situada a 7-9 m. sobre el cauce actual, está formada por gravas fuertemente encostradas por carbonatos que se corresponden con una de las etapas cuaternarias de fuerte acumulación fluvial, que en el cercano río Gállego ha sido datada en 156-181 ka BP por Benito et al. (1998) y Benito et al.
(2010). El grosor de estos depósitos impide que los yesos infrayacentes aparezcan en superficie en el antiguo ámbito urbano. Por otra parte, una terraza más reciente, de edad holocena, se extiende por el margen septentrional del río Ebro, en el actual barrio del Actur y por el sector noreste de la ciudad romana (Peña-Monné et al., 2013). Esta misma terraza aparece en las excavaciones realizadas en diversos puntos de la ciudad, en muchos casos, contemporánea con el desarrollo de la ciudad romana.

\section{Objetivos y metodología}

El objetivo principal del trabajo es aportar nuevos datos al conocimiento de la topografía y morfología 
del terreno ocupado por la ciudad romana de Caesar Augusta. Su consecución pasa por lograr varios objetivos secundarios: en primer lugar, poner en valor documentos cartográfícos históricos como información básica o de consulta para este tipo de trabajos y, en segundo lugar, mostrar la capacidad de los Sistemas de Información Geográfica (SIG) para la reconstrucción de topografías originales y su interpretación. La base del trabajo es la utilización de cartografía histórica y su incorporación y manejo en el entorno del software ArcGIS Desktop 10.0 y sus módulos ArcMap y ArcScene.

La utilización de mapas antiguos para la reconstrucción de paisajes o topografías no es una técnica muy extendida, fundamentalmente por la escasez de documentos cartográficos con información topográfica y, más aún, con un nivel de precisión apropiado. Sin embargo, existen algunos trabajos sobre ciudades españolas como es el caso de Tarragona (Gabriel Costa, 2001; Orengo et al., 2011), donde se ha combinado la información de cartografía histórica con documentación arqueológica y fotografías de archivo. Por su parte, los Sistemas de Información Geográfica (SIG) fueron adoptados casi desde su aparición para la elaboración de reconstrucciones arqueológicas, en especial de edificios y paisajes sobre la topografía actual, aunque son pocos los trabajos que abordan el aspecto de la reconstrucción topográfica en ámbitos urbanos, destacando en este sentido los trabajos de Fiz Fernández (2001) y Fiz y

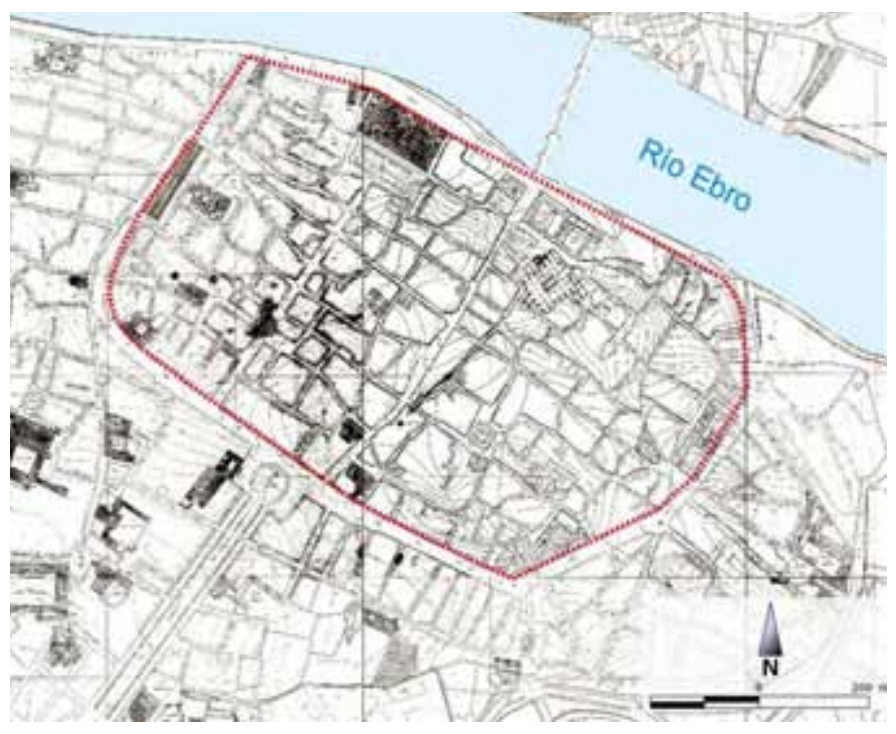

Figura 4. Detalle del plano de Casañal en el que se ha señalado como referencia el límite del recinto de la muralla romana.

Figure 4. Detail of Casañal s map with the enclosure of the Roman wall marked.
Macías (2007) para la ciudad romana de Tarraco, donde ponen de manifiesto no sólo la distribución de los edificios públicos en este periodo histórico, sino también su relación con la morfología del terreno e, incluso, analizan planteamientos ideológicos derivados de la planificación de la urbe y en relación con las vías de entrada a la ciudad.

En nuestro caso, se contó con una imagen de alta resolución del plano original de Dionisio Casañal de 1880, aportada por el Ayuntamiento de Zaragoza, a 1200 pixeles por pulgada de resolución y en 20 fragmentos (Fig. 4), que fue incorporada en la geodatabase generada para desarrollar el proyecto en un entorno SIG. Una vez incorporada la imagen, fue georeferenciada mediante la búsqueda de puntos de control coincidentes entre el mapa (datos de origen) y la ortofotografía de 2012 (datos de destino) en base al plano de la ciudad, en el sistema de coordenadas ETRS89 huso 30 zona Norte, para cada uno de los fragmentos por separado.

En el proceso de georreferenciación se utilizaron al menos 10 puntos de control por fragmento y se tuvieron en cuenta los valores de Error Cuadrático Medio (RMSE) resultantes del proceso, finalizando éste cuando dichos valores se situaban próximos a " 0 ". Sin embargo, como un valor de RMSE no significa obtener un registro preciso, se comprobó visualmente la coincidencia de puntos comunes entre el mapa y ortofotografía, incorporando un número mayor de puntos cuando se observaban superposiciones con errores de coincidencia superiores a $1 \mathrm{~m}$.

Una vez incorporadas y posicionadas correctamente las imágenes se procedió a la digitalización de las curvas de nivel presentes en el mapa de Dionisio Casañal, vectorizando de esta manera la información topográfica con equidistancia de medio metro, obteniendo el fichero necesario para poder elaborar a través de la creación de una red de triangulación irreguIar (TIN) el correspondiente Modelo Digital de Terreno (MDT) (Fig. 7).

Con el MDT disponible, se añadió a esta información todos los datos conocidos y con referencia espacial procedentes de las excavaciones arqueológicas, en especial el trazado de la muralla, teatro romano, foro, termas y cloacas (Fig. 7), trasladando posteriormente esta información al módulo ArcScene de ESRI, con el que se procedió a generar los modelos tridimensionales (Fig. 8). Estos han permitido visualizar en 3D la reconstrucción del espacio ocupado por la antigua ciudad de Caesar Augusta y el entorno próximo, facilitando la interpretación y el análisis de la topografía obtenida.

Aunque el elemento principal de reconstrucción es la cartografía histórica, también se ha utilizado infor- 
mación geomorfológica de la ciudad y su entorno, basada en la realización de cartografías geomorfológicas detalladas, dataciones radiométricas y estudios geoarqueológicos para reconocer las principales etapas evolutivas del Holoceno Superior (Ángeles et al., 2004; Peña-Monné et al., 2000, 2004; Constante et al, 2010, 2011; Peña-Monné, 2013; Pérez Lambán et al., 2014). En este sentido, también han sido de especial relevancia las cotas obtenidas en las sucesivas excavaciones arqueológicas en la ciudad (Escudero and Galve, 2014), cuyos datos se han utilizado para la validación del modelo y la reconstrucción de un perfil topográfico aproximado de la ciudad de Sur a Norte, pasando por los principales edificios conocidos como el teatro romano, los restos de murallas romana y medieval y las tres unidades morfológicas (terraza antigua de gravas, nivel de arenas superior y terraza reciente) que caracterizan el sector oriental de la ciudad.

\section{Resultados}

Una vez creado el MDT a partir de las curvas de nivel digitalizadas del mapa de Casañal, se ha realizado un análisis espacial de la variación de las cotas altitudinales de la ciudad romana respecto a la actual (Fig. 5), cuyos datos se han obtenido del MDT05 Lidar del Instituto Geográfico Nacional. Una vez realizada la comparación de los dos MDTs, se puede observar como el solar ocupado por Caesar Augusta concentra el mayor número de zonas donde la variación de altitud ha sido negativa respecto a la situación actual, poniendo de manifiesto como las grandes transfor-

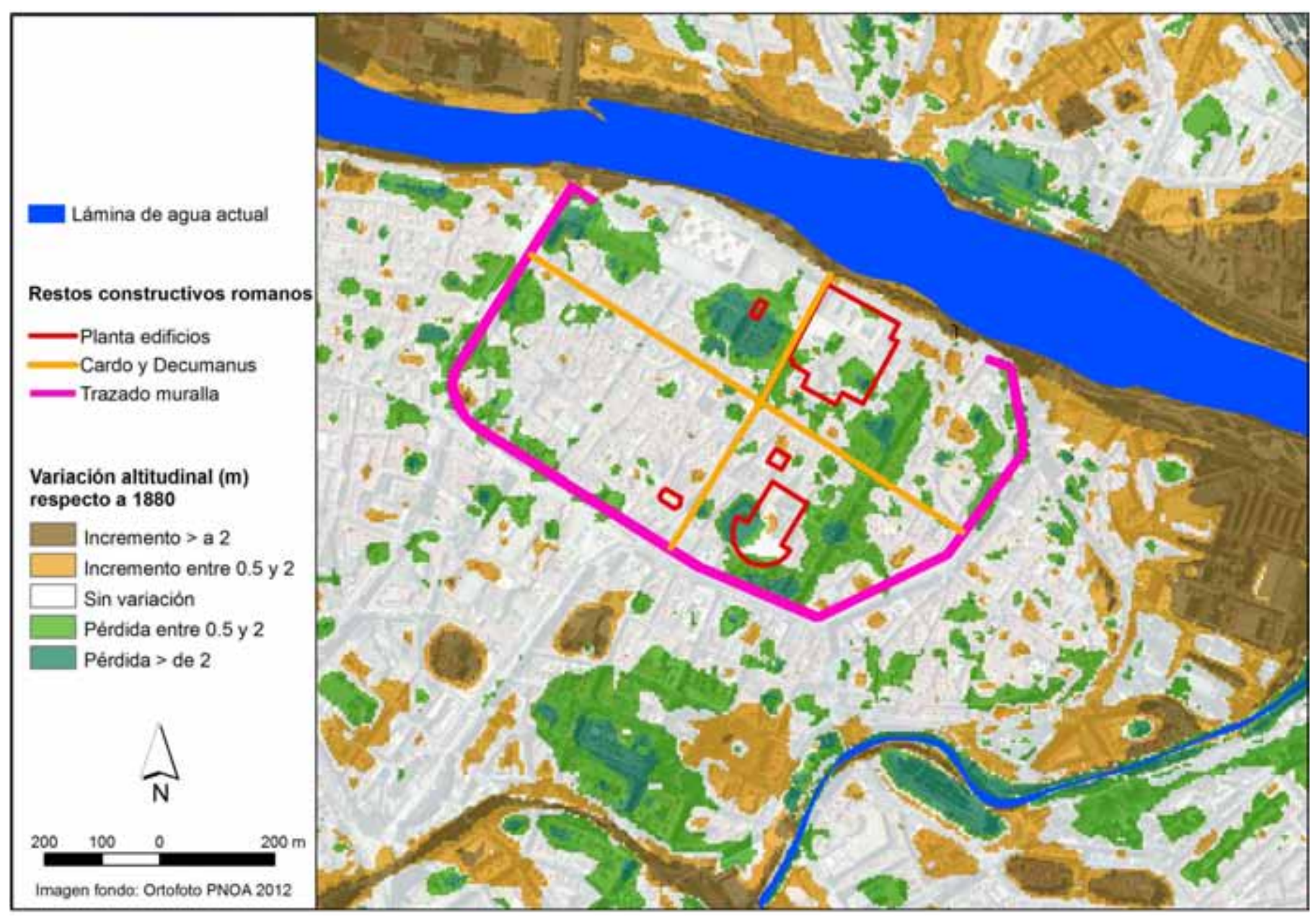

Figura 5. Diferencias altitudinales entre el modelo elaborado a partir del mapa de Casañal y el MDT5 LiDAR del Instituto Geográfico Nacional.

Figure 5. Altitudinal differences between the DEM derived Casañal s map and the MDT5 LiDAR (National Geographic Institute). 
maciones de la ciudad anteriormente citadas, afectaron especialmente a la topografía del casco antiguo.

Estas modificaciones han supuesto variaciones de las cotas de la topografía de más de $1 \mathrm{~m}$ y, en algunas zonas, incluso de más de dos $2 \mathrm{~m}$ de diferencia. Estos puntos se concentran especialmente en el entorno del teatro romano, en el margen oeste de la muralla a lo largo de todo su trazado en este sector $y$, sobre todo, a lo largo de la calle San Vicente de Paul y el sector de la Plaza del Pilar junto al Ayuntamiento y la Plaza de la Seo (Figs. 5 y 6).

Los cambios sugeridos por estas variaciones de las cotas del relieve original han supuesto la disminución de la rugosidad de la topografía, de tal forma que el conjunto formado por elevaciones y vallonadas con orientación NNE-SSO, que son evidentes en el modelo de 1880 (Figs. 6 y 7) en la actualidad han perdido entidad. No obstante, la disposición de las citadas vallonadas se intuye a través de las principales calles vertebradoras del casco antiguo de la ciudad, como la avenida Cesar Augusto y calles Alfonso I, Don Jaime I y Gavín, que mantienen su trazado, disminuyendo su pendiente longitudinal en dirección transversal al cauce del río Ebro, que se ubicaría aproximadamente a $189 \mathrm{~m}$ sobre el nivel del mar, pudiendo ser considerada esta cota como el nivel de base medio de estas zonas de drenaje natural de la ciudad romana.

Por su parte, las morfologías más elevadas constituyen relieves con litologías detríticas cuaternarias, gravas y arenas fluviales, como se viene observando en las excavaciones arqueológicas o en los solares en construcción de la ciudad. En ellos es frecuente la aparición de gravas fuertemente cementadas, que en

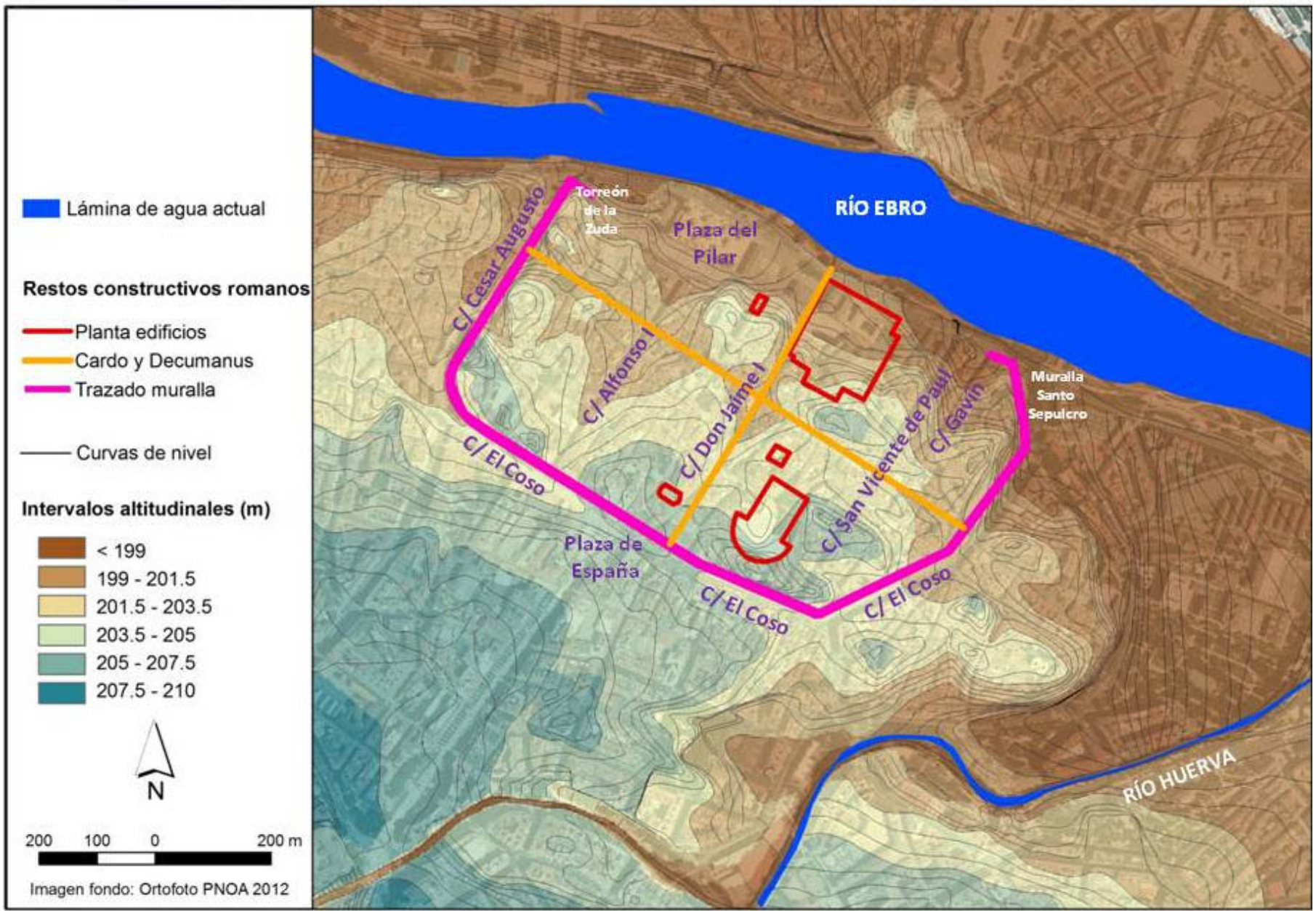

Figura 6. MDT y curvas de nivel de Casañal con las principales calles de referencia y principales restos constructivos romanos de la ciudad.

Figure 6. DEM and contour lines of Casañal s map with the main streets and main constructive Roman remains indicated. 


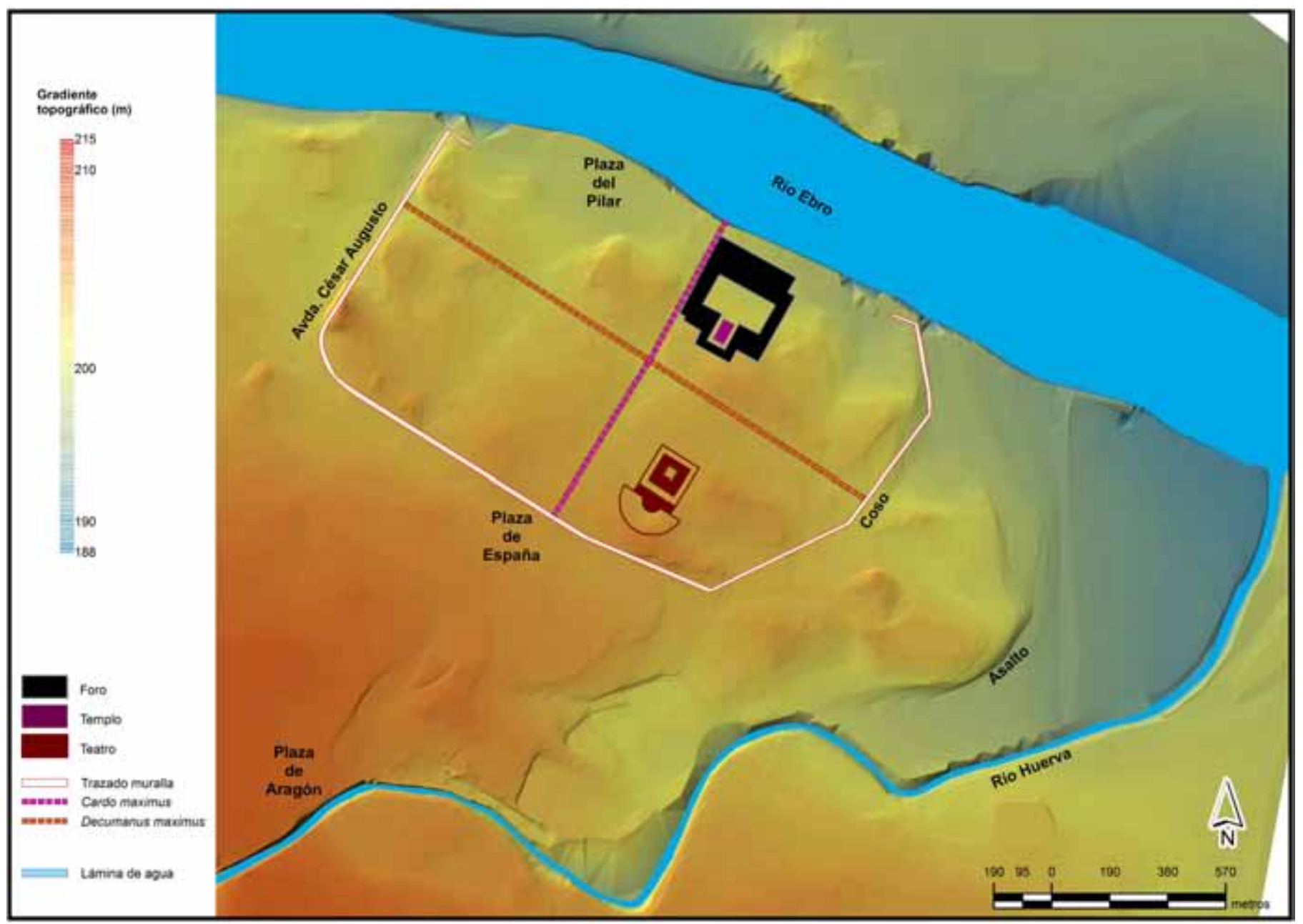

Figura 7. MDT del antiguo casco urbano de Zaragoza obtenido del mapa topográfico de Casañal (1880) mostrando algunos de los restos romanos más significativos de Caesar Augusta.

Figure 7. DEM of the historical centre of Zaragoza from the topographic map of Casañal (1880) showing some of the significant Roman remains of Caesar Augusta.

ocasiones sirven como base de la cimentación romana, lo que ha podido resultar decisivo en su conservación como puntos elevados destacados del resto a lo largo del tiempo.

En detalle, podemos observar la disposición singular de algunas de las morfologías que se extraen del MDT del mapa de Casañal. Es posible definir hasta un total de cuatro conjuntos de elevaciones en el espacio ocupado por la ciudad romana, delimitadas por cinco vallonadas o surcos de orientación NNE-SSO (Figs. 6 y 7). Las zonas más elevadas presentan alturas comprendidas entre los 204 y 208 m, destacando entre 3 y $4 \mathrm{~m}$ sobre las zonas más bajas que las limitan.

El desnivel que muestran algunos de estos conjuntos es especialmente significativo en el sector noroccidental, donde alcanza los $4 \mathrm{~m}$ entre el margen de la ciudad romana y la vaguada alargada que se observan al pie del trazado de la muralla (Figs. 8A, 8C). Este sector presenta, además, en la confluencia de las actuales calles Cesar Augusto y Coso, la prolongación del surco tras dibujar un ángulo de $90^{\circ}$, que resalta respecto a la distribución general del relieve. Esta situación se repite a lo largo del trazado de la calle Coso, por lo que se plantea la hipótesis de que esta vallonada sea un foso defensivo creado artificialmente en su mayor parte, con la finalidad de incrementar la función defensiva de la muralla y que rodearía la ciudad por dos de sus lados.

Por otra parte, frente al dispositivo general NNESSW, existe un espacio de disposición transversal y rectilíneo que coincide con el trazado Decumanus maximus de la ciudad romana. Esta vía muestra en el plano de Casañal una topografía ondulada, atrave- 
sando los conjuntos elevados anteriormente citados (Figs. 8A, 8C), por lo que parece que fue construida aplanando en parte la topografía inicial.

En el entorno del actual Teatro Principal se encuentra un conjunto de elevaciones naturales que fueron aprovechadas para, con pequeñas modificaciones, instalar el graderío del teatro romano, que se apoya sobre una formación arenosa del río Huerva, depositada sobre la terraza fluvial antigua del Ebro, anteriormente mencionada. Igualmente, muestra un desnivel de $3 \mathrm{~m}$ sobre el Coso, posiblemente creado por la apertura del foso anteriormente apuntado, marginando la muralla en todo este sector meridional (Fig. 8D).

Fuera del recinto amurallado, entre el Coso Bajo y el cauce del río Huerva, la ciudad romana se amplió en época tardía. En este sector se observa otro conjunto elevado irregular que resalta entre 2 y $2,5 \mathrm{~m}$ sobre el Coso y que pierde altura escalonadamente hacia el curso del río Huerva, que se encaja en este sector en una terraza baja (Fig. 8B). La pendiente resultante de esta disposición topográfica fue aprovechada para el trazado de las cloacas de esta zona de ampliación de la ciudad, que vertía sus residuos al río Huerva en lugar de al Ebro, como el resto del sistema ubicado dentro del recinto amurallado (Escudero and Galve, 2013).

Para complementar la información cartográfica anterior se ha elaborado un perfil topográfico siguiendo el trazado del Decumanus $I X$ que permite apreciar la topografía de 1880 (Fig. 9a), con las irregularidades todavía existentes en el terreno. Se apre-
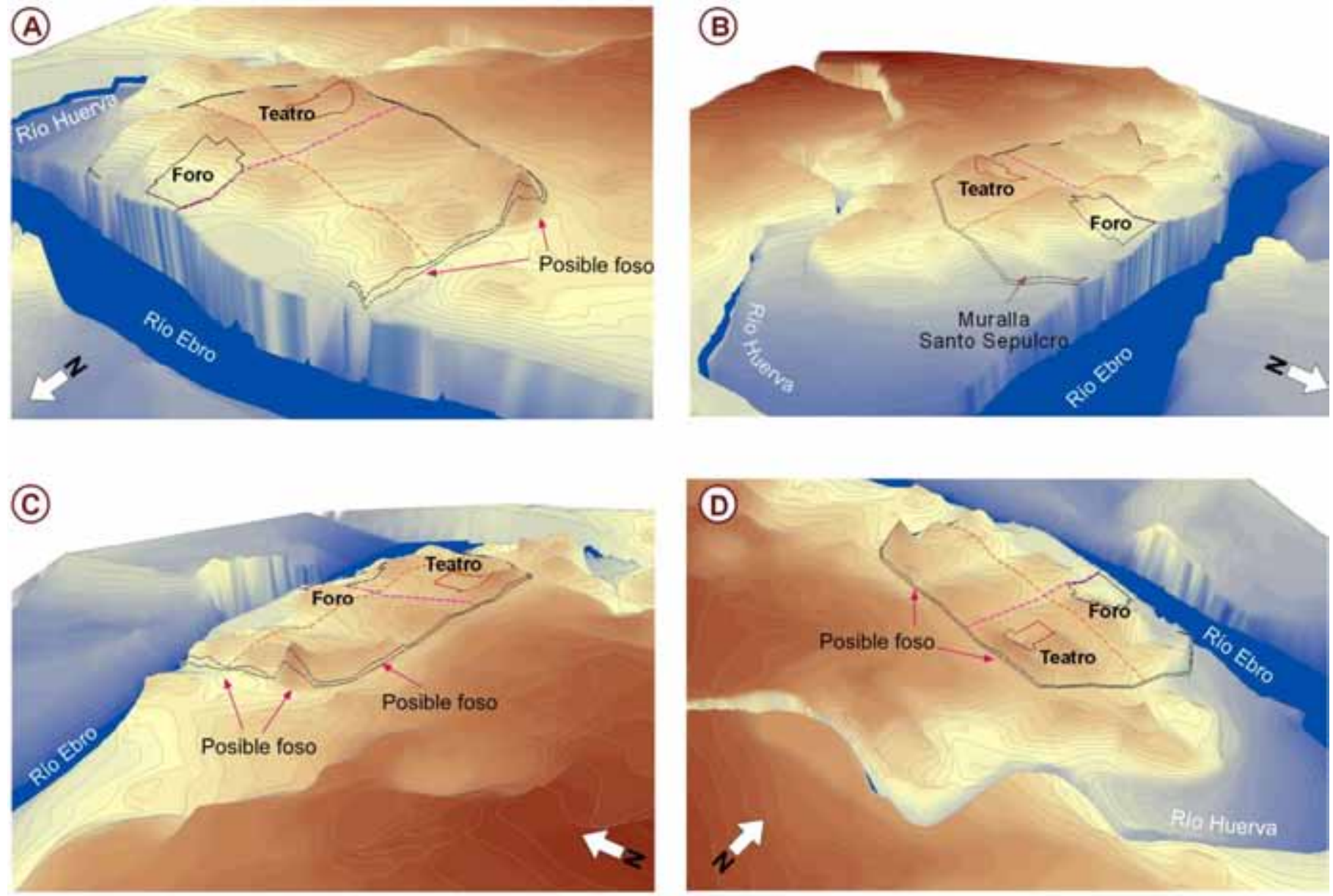

\section{zza: Muralla romana -.. Decumanus maximus -.- Cardo maximus}

Figura 8. Cuatro perspectivas diferentes en 3D del MDT de la ciudad de Zaragoza a partir del mapa de Casañal. La flecha indica la posición del Norte.

Figure 8. Four different $3 D$ views of the DEM of the Zaragoza city from Casañal's map. The arrow indicates the position of the north. 
cia también la posición de las murallas oeste y este, ésta última acompañada por un foso, aunque también se ha considerado la posibilidad de que el río Huerva tuviera su curso bajo ocupando este sector, circulando pegado a la muralla en un primer momento, antes de ocuparse la zona a extramuros en Época Romana más tardía tras desviar este río hacia el este. También se observa la posición de la muralla medieval en este mismo sector. Un segundo perfil se ha trazado a través del teatro y del foro romanos (Fig. 9b) mostrando el desnivel sobre el río Ebro y el foso que limita la muralla romana por el NNW, así como la adaptación delTeatro a la topografía y del Foro al desnivel entre la terraza antigua y la terraza holocena.

La información geoarqueológica y las cotas derivada de las intervenciones arqueológicas (Escudero and Galve, 2014), han permitido reconstruir la disposición en el sector oriental de la ciudad de las tres unidades sedimentarias fluviales que caracterizan el solar de la ciudad romana (Fig. 10). Un conjunto de gravas fuertemente cementadas constituyen la unidad basal, que corresponden a una de las terrazas antiguas del río Ebro, que por su correlación con la terraza equivalente del río Gállego, se situaría en edades en torno a 156-180 ka BP (Benito et al., 2010). Por encima, aparece una cubierta de arenas, a alturas de unos 200 m s.n.m., pertenecientes a inundaciones holocenas del río Huerva que se prolongan por los niveles analizados por Peña-Monné et al. (2004) en el curso bajo de dicho río. En las cotas inferiores aparecen gravas de inundación de época reciente cuya edad es posterior al $100 \mathrm{dC}$ (Constante, 2009; PeñaMonné et al., 2009), junto a la base de la muralla del Santo Sepulcro, formando un escalón más bajo que fue ocupado en la ampliación de la ciudad romana y que sufría las crecidas en época romana (Constante, 2009; Peña-Monné et al., 2009).

En cuanto a la morfología fluvial de los dos cauces principales que rodean la ciudad, el río Ebro se mantuvo en una posición similar hasta el inicio de la Pequeña Edad del Hielo, en que las grandes crecidas harán desaparecer las vías romanas del entorno de la ciudad y algunos núcleos de población ubicadas siguiendo los primeros miliarios de su trazado. Destaca la crecida de 1380 , momento en que se crea el cauce denominado de las "Balsas de Ebro Viejo", con grave peligro para la ciudad, así como las grandes riadas producidas durante todo el siglo XVIII (Peña-Monné et al., 2013).

Pese a este periodo de mayor inestabilidad, el cauce actual mantiene su mismo trazado en la ciudad, siendo su puente principal (actual Puente de Piedra), pese a las sucesivas reconstrucciones, el único paso posible en este sector del valle. Esta situación estable

\section{a) \\ w} CAESAR AUGUSTA

rio Ebro

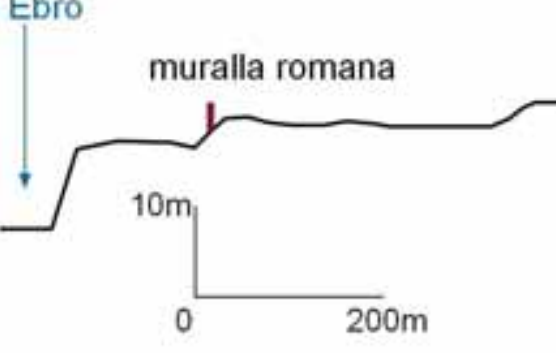

b)

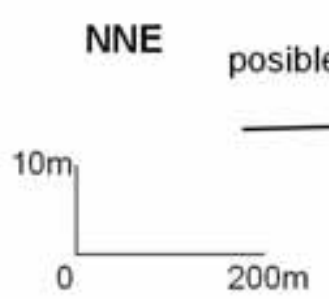

Decumanus IX

muralla rod 

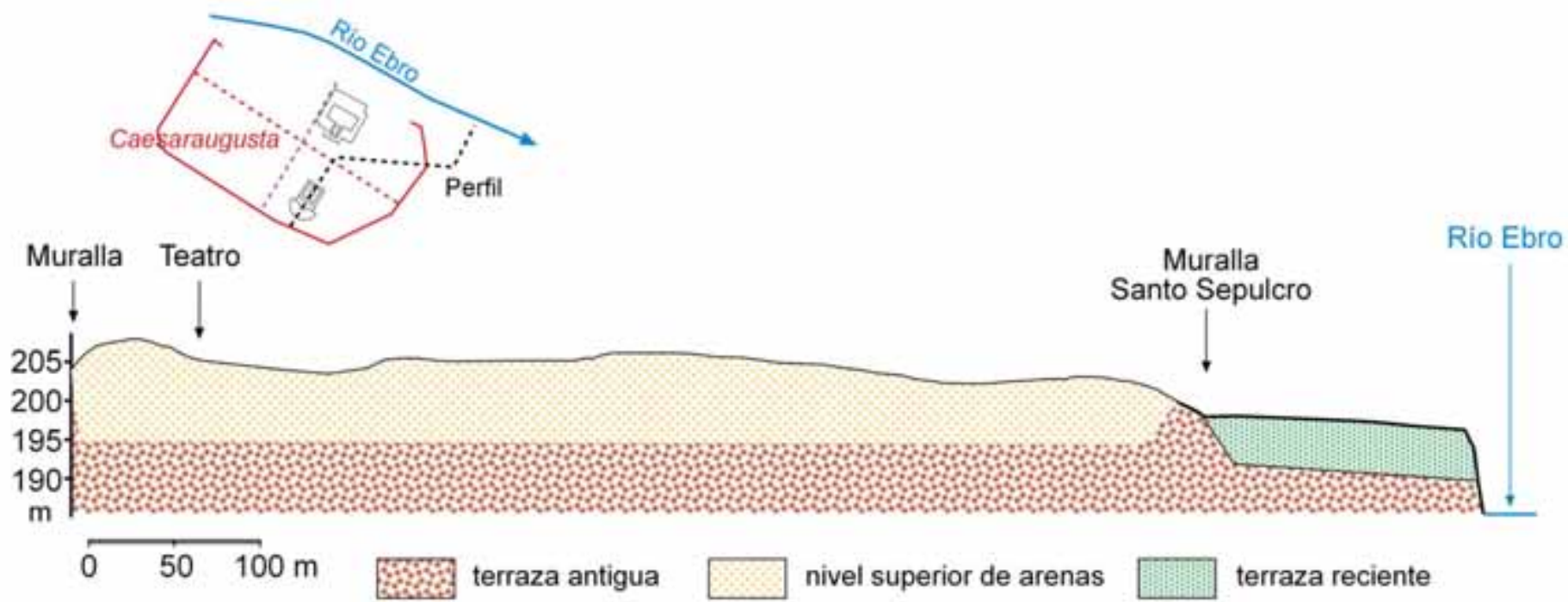

Figura 10. Reconstrucción de las unidades sedimentarias en las que se apoya la ciudad romana entre el sector Sur de la muralla y el río Ebro, atravesando la ubicación del teatro romano y la muralla del Santo Sepulcro.

Figure 10. Reconstruction of the sedimentary units where the Roman city was located, from the southern stretch of the wall, across the Roman theatre, to the Holy Sepulchre stretch of the wall.

puede estar también relacionada con la presencia de varios elementos geomorfológicos favorables. En primer lugar, la cercana confluencia con el río Gállego, importante afluente pirenaico cuyos registros sedimentarios hablan de su capacidad de aporte de material al cauce principal, por lo que su cono de expansión de sedimentos seguramente favoreció el mantenimiento del cauce del Ebro en su parte meridional una vez superada la ciudad. Por otra parte, la existencia de una morfología de origen kárstico denominada popularmente "pozo de San Lázaro", inmediatamente al Este del Puente de Piedra, ha podido estar actuando ya en época romana como el punto más bajo del cauce y dirigir, por tanto, la corriente principal del río. Por su parte, el cauce del río Huerva presenta una morfología bien distinta, alcanzando la zona urbana después de atravesar un estrecho y profundo cañón fluvial. Hoy esta morfología no es apreciable, ya que fue cubierto durante las obras de ensanche de la ciudad del siglo XIX, pero queda bien dibujado en los modelos derivados del mapa de Casañal (Fig. 8D). Este pequeño cañón fluvial alcanza una profundidad superior a los $7 \mathrm{~m}$ en algunos puntos, con anchuras que no superan los $5 \mathrm{~m}$, debido a la resistencia de las terrazas cuaternarias del río Huerva al desarrollo de la erosión lateral, al tiempo que la dinámica de incisión se convirtió en dominante con posterioridad a la Época Romana (siglos V-VII, según Peña-Monné et al. 2004). Esta incisión se generó sobre los depósitos holocenos resultantes de la intensa erosión producida en su cuenca baja debido a la intervención humana desde el Neolítico hasta la Época Romana tardía, que determinó la desaparición de los suelos y la cubierta vegetal que protegía las laderas del valle hasta entonces, tanto en el río Huerva (Peña-Monné et al., 2004; Pérez Lambán et al., 2014) como en el Ebro (Constante et al., 2009, 2010). Como hemos indicado, cabría la posibilidad de que el trazado final del río Huerva fuera desviado, ya que hay registros sedimentarios de este río situados por debajo de la muralla romana del sector del Santo Sepulcro, que fueron datados $\operatorname{con}{ }^{14} \mathrm{C}$ en la Edad del Bronce $(1168 \pm 86 \mathrm{aC})$ y contienen, además, restos de cerámica de esa misma época (Peña-Monné et al., 2009). Por otra parte, la aparición de campos de ánforas instalados en Época Romana (siglo I-II dC) para elevar el terreno en este mismo sector (Peña-Monné et al., 2009) y en la plaza de San Miguel, pudieran ser testimonios de una elevación del terreno para ampliar la ciudad empujando al río hacia su posición actual, de la que no volverá a salir debido a su posterior incisión, excepto en eventos extraordinarios en que las crecidas ocuparon la parte sur de la ciudad de Zaragoza.

\section{Conclusiones}

Los modelos tridimensionales, obtenidos a partir del mapa topográfico de Casañal de 1880, nos han permitido aproximarnos a la topografía original de la ciudad romana de Caesar Augusta, a pesar de la separa- 
ción temporal existente. Aunque este modelo solo es válido para una reconstrucción general, si que ha mejorado nuestro conocimiento sobre el trazado de la topografía urbana y la adaptación de determinados edificios públicos, como el Teatro, el Foro o la muralla romana a los accidentes del terreno. El trazado de la red urbana y del sistema de alcantarillado también quedó condicionado en gran medida por la disposición del relieve.

La observación detallada de las depresiones que bordean por el sur la muralla de la ciudad, plantea que podrían constituir un foso defensivo de la misma y que, por lo tanto, tendrían un origen antrópico, con toda probabilidad de época romana, cuya finalidad sería dotar de mayor capacidad defensiva a las partes de la ciudad no rodeadas por ríos, ya que constituían los principales puntos de debilidad. Este mismo dispositivo defensivo probablemente se mantuvo $y$ reforzó durante el periodo islámico, lo que ha debido contribuir a que sea todavía identificable en la topografía de Casañal.

La integración de cartografía histórica como la utilizada para Caesar Augusta en herramientas informáticas apropiadas, como en este caso el software ArcGIS, y la utilización de técnicas de georeferenciación adecuadas, han demostrado su aplicabilidad a la reconstrucción de áreas urbanas antiguas en las que las transformaciones históricas sufridas han hecho desaparecer los rasgos topográficos originales.

\section{Referencias}

Álvarez Gracia, A. 1985. Topografía antigua de la ciudad de Zaragoza. Ayuntamiento de Zaragoza, Zaragoza. 35 pp.

Ángeles, G., J.L. Peña-Monné, J.L. and Longares, L.A. 2004. Cartografías de reconstrucción paleoambiental y riesgo geomorfológico del Holoceno superior en el valle del Río Huerva (Depresión del Ebro). In: Peña-Monné, J.L., Longares, L.A. and Sánchez, M. (eds.), Geografía Física de Aragón. Aspectos generales y temáticos. Universidad de Zaragoza e Institución Fernando el Católico, Zaragoza, 303-314.

Arce, J., 2001. Caesaraugusta, ciudad romana. Guara Editorial, Zaragoza.

Beltrán Lloris, F. (Ed.) 2007. Zaragoza. Colonia Caesar Augusta. In: Ciudades romanas de Hispania. Las capitales provinciales, 4. L'Erma di Bretschneider, Roma,

Beltrán Lloris, M. and Fatás, G. 1998. Historia de Zaragoza, vol. 2, César Augusta, ciudad romana. Zaragoza. Ayuntamiento-Caja de Ahorros de la Inmaculada, Zaragoza.

Benito, G., Pérez-González, A., Gutiérrez, F. and Machado, M.J. 1998. River response to Quaternary subsidence due to evaporite solution (Gállego River, Ebro Basin, Spain). Geomorphology, 22 (2), 243-263.
Benito, G., Sancho, C., Peña-Monné, J.L., Machado, M.J. and Rhodes, E. 2010. Large-scale karst subsidence and accelerated fluvial aggradation during MIS6 in NE Spain: Climate and paleohydrological implications. Quaternary Science Reviews, 29, 2694-2704.

Betrán, R. and Serrano, L. 2014. La Zaragoza de 1908 y el plano de Dionisio Casañal. La construcción de una ciudad burguesa. Institución Fernando EI Católico, Zaragoza, $191 \mathrm{pp}$.

Constante, A. 2009. Estudio geoarqueológico de los registros holocenos del sector central del valle del Ebro. Tesis doctoral Universidad de Zaragoza, Zaragoza.

Constante, A., Dossche, R., Peña-Monné, J.L., Sancho, C. and de Dapper, M. 2009. Holocene evolution and geoarchaeology in the Ebro valley around Zaragoza (Northern Spain). In: De Dapper, M., Vermeulen, F., Deprez, S. and Taelman, D. (eds.): OI man river Geo-archaeological aspects of rivers and river plains. Academia Press, Ghent, 241-256.

Constante, A., Peña-Monné, J.L. and Muñoz, A. 2010. Alluvial geoarchaeology of an ephemeral stream: Implications for Holocene landscape change in the Central part of the Ebro Depression, Northeast Spain. Geoarchaeology, 25 (4), 475-496.

Constante, A., Peña-Monné, J.L., Muñoz, A. and Picazo, J. 2011. Climate and anthropogenic factors affecting alluvial fan development during the Late Holocene in the Central Ebro valley, Northeast Spain. Holocene, 21, 275286.

Clemente Fernández, E. 1996. La transformación de la ciudad de Zaragoza en el siglo XIX. In: Historia de Aragón. Economía y sociedad, Tomo II. Institución Fernando el Católico, Zaragoza, 431-444

Escudero, F. and Galve, M.P. 2013. Las cloacas de Caesar Augusta y elementos de urbanismo y topografía de la ciudad antigua. Institución Fernando El Católico, Zaragoza, 492 pp.

Fatás, G. (dir.) 2008. Guía Histórico-Artística de Zaragoza. Institución Fernando el Católico-Ayuntamiento de Zaragoza, Zaragoza.

Faus Pujol, M.C. 1978. El ferrocarril y la evolución urbana de Zaragoza. Cuadernos de Zaragoza 33, 83-114

Fiz Fernández, I. 2001. Usos de un SIG, Sistema de Información Geográfico, en la construcción de una Planimetría Arqueológica para Tarragona (I). Revista d'Arqueológia de Ponent, 11/12,111-121.

Fiz Fernández, I. and Macias, J.M. 2007. Forma Tarraconis? GIS use form urban archaeology. In: Figueiredo, A. and Velho, G. (eds.), The World is in your eyes, Proceedings of the XXXIII Computer Applications and Quantitative Methods in Archaeology Conference. Tomar, 423-427.

Gabriel Costa, R. 2001. Aproximación a la topografía antiga de Tarragona. Bulletí arqueològic, 23, 281-345.

Galve Izquierdo, P. 2004. Una ciudad consolidada: Caesaragusta a mediados del siglo I. In: Álvarez, A. (coord.), Zaragoza. Visiones de una ciudad. ArchivoBiblioteca-Hemeroteca, Ayuntamiento de Zaragoza, Zaragoza.

Germán Zubero, L. 1996. La transformación de la ciudad. Zaragoza en el siglo XX (1900-1936). In: Historia de 
Aragón. Economía y sociedad, Tomo II. Institución Fernando el Católico, Zaragoza, 459-468.

Lostal, J. and Ansón, A. 2001. Historia de cuatro ciudades: Salduie, Caesaraugusta, Saraqusta, Zaragoza. Ayuntamiento-Caja Inmaculada, Zaragoza.

Orengo, H.A., Fiz, J.I. and Macías, J.M. 2011. Restitución 3D de la topografía de la antigua ciudad de Tarraco en un entorno SIG: propuestas metodológicas y primeros resultados. In: Mayoral, V. and Celestino, S. (eds.), Tecnologías de información geográfica $y$ análisis arqueológico del territorio: Actas del V Simposio Internacional de Arqueología de Mérida. Anejos del Archivo Español de Arqueología. LIX. CSIC, Madrid, 713722.

Paz Peralta, J.A., 2015. Los cubos de las murallas de Zaragoza y del palacio de la Aljaferia (1065-1075). Paradigmas de la arquitectura militar en Al-Andalus. Caesaraugusta, 84, 5-328.

Peña-Monné, J.L. 2013. Methodologhical proposals for landscape evolution study around archaeological sites in semiarid regions. The Caesar Augusta-SaraqustaZaragoza (Spain) example. In: Vermeulen, F. and Corsi, C. (eds.), Non-destructive approaches to complex archaeological sites in Europe: around-up. Radio-Past Colloqium, Ghent University, 22-23.

Peña-Monné, J.L., Echeverría, M.T., Chueca, J. and Julián, A. 2000. Processus d accumulation et $d$ incision pendant I Antiquité Classique dans la vallée de la Huerva (Bassin de I Ebre, Espagne). In: Vermeulen, F. (ed.), Geoarchaeology of the Landscapes of Classical Antiquity. Leuven, 151-159.

Peña-Monné, J.L., Escudero, F., Rubio, V., Constante, A. and Pellicer, F. 2009. Geoarchaeological contributions concerning the Roman city wall of Caesar Augusta in the sector of the Santo Sepulcro (Zaragoza, Spain). In: De Dapper, M., Vermeulen, F., Deprez, S. and Taelman, D. (eds.), OI man river Geo-archaeological aspects of rivers and river plains. Academia Press, Ghent. 541-551.

Peña-Monné, J.L., Julián, A., Chueca, J., M.T. Echeverría, M.T. and G. Ángeles, G. 2004. Etapas de evolución holocena en el valle del río Huerva: Geomorfología y Geoarqueología. In: Peña-Monné, J.L., Longares, L.A., and Sánchez, M. (eds.), Geografía Física de Aragón. Aspectos generales y temáticos. Universidad de Zaragoza e Institución Fernando el Católico, Zaragoza, 289-302.

Peña-Monné, J.L., Longares, L.A. and Constante, A. 2013. El marco geográfico de Caesar Augusta. In: Escudero, F. and Galve, M.P. (eds.), Las cloacas de Caesar Augusta y elementos de urbanismo y topografía de la ciudad antigua. Institución Fernando el Católico, Zaragoza, 33-47.

Peña-Monné, J.L., Rubio, V., Longares, L.A., Gutiérrez, F.J. and Constante, A. 2011. El castillo medieval de Juslibol (Zaragoza). Datos preliminares para una reconstrucción geoarqueológica. In:Turu, V, and Constante, A. (eds.), EI Cuaternario en España y áreas afines. Avances en 2011. XIII Reunión Nac. del Cuaternario, Andorra la Vella, 213215

Pérez-Lambán, F., Peña-Monné, J.L., Fanlo, J., Picazo, J.V., Badia, D., Rubio. V., García-Jiménez, R. and Sampietro, M.M. 2014. Paleoenvironmental and geoarchaeological reconstruction from late Holocene slope records (Lower Huerva Valley, Ebro Basin, NE Spain). Quaternary Research, 81, 1-14.

Quirantes, J. 1978. Estudio sedimentológico y estratigráfico del Terciario continental de los Monegros. Institución Fernando el Católico, CSIC, Zaragoza, 234 pp.

Villanova Valero, J. L. 2011. Dionisio Casañal y Zapatero: Del catastro a la topografía (1864-1878). In: Montaner, C., Nadal, F. and Urteaga, L. (eds.), Cartografia i agrimensura a Catalunya i Balears (segles $X I X-X X)$. Institut Cartogràfic de Catalunya, Barcelona, 209-223.

Recibido: marzo 2015

Revisado: junio 2015

Aceptado: junio 2015

Publicado: marzo 2018 
